\title{
Nasofrontal Mucocele Following after Craniotomy Procedure
}

\author{
Tommy Rizky Hutagalung ${ }^{1}$, Abdurrahman Mouza ${ }^{1}$ \\ ${ }^{1}$ Department of Neurosurgery, Universitas Sumatera Utara, Medan, Indonesia
}

\begin{abstract}
Introduction : Mucocele is a chronic, expanding, mucosa-lined lesion of the paranasal sinus characterized by mucous retention that can be infected becoming a mucopyocele. They originate from obstruction of the sinus ostium by congenital anomalies, infection, inflammation, allergy, trauma (including surgery) or a benign or malignant tumor. The frontal sinuses are most commonly affected, and subsequently ethmoidal sinuses.

Case Report : A 56 years old man, presented with a lump on the left and right forehead accompanied by a protruding left eye since 6 months and is getting wors. Patient with a history of craniectomy debridement surgery indicated for open depressed fracture due to an accident 12 years ago, then underwent a titanium mesh cranioplasty 11 years ago. From examination of the head CT scan revealed a solid mass lesion filling the left and right frontal sinuses expands into the left orbital cavity. Bifrontal craniotomy was performed on the patient. Discussion : Mucoceles are mucous-secreting expansive pseudocystic formations, and capable of expansion by virtue of a dynamic process of bone resorption and new bone formation. They result from obstruction of a sinus ostium and frequently are related to a previous condition as chronic sinusitis, trauma, surgery or expansible lesion. With continued secretion and accumulation mucus, the increasing pressure causes atrophy or erosion of the bone of the sinus, allowing the mucocele to expand in the path of less resistance. This may be into the orbit, adjacent sinuses, nasal cavity, intracranial or through the skin; intracranial and orbital extension were demonstrated in this patient.

Conclusion : Frontal mucoceles are benign and curable, but early diagnosis and treatment of them is important. Open surgery remains a valid procedure in frontal mucoceles with orbital and/or intracranial extension and in cases where the district anatomy is unfavourable for a purely endonasal approach.
\end{abstract}

Keyword : Mucocele, Frontal, Infection, Neurosurgery

\section{Introduction}

Mucocele is a chronic, expanding, mucosa-lined lesion of the paranasal sinus characterized by mucous retention that can be infected becoming a mucopyocele. They originate from obstruction of the sinus ostium by congenital anomalies, infection, inflammation, allergy, trauma (including surgery) or a benign or malignant tumor. Even benign, they have a tendency to expand by eroding the surrounding bony walls that displaces and destroys structures by pressure and bony resorption. [1]

Regardless of the reconstructive technique used, a small percentage of patients can be expected to develop frontal sinus mucoceles after frontal craniotomy. There is also demonstrates the wide range in time to mucocele development and the need for longterm surveillance after craniotomy procedures. [2] 
The frontal sinuses are most commonly affected, and subsequently ethmoidal sinuses. Symptoms and signs of frontal mucoceles include pain, swelling, exophtalmos, diplopia and loss of vision. An erosive mucocele may lead to meningitis, meningoencephalitis, pneumocephalus, brain abscess, seizures or Cerebro-Spinal Fluid (CSF) fistulas. They are benign lesions that occur with similar frequency in adults of both sexes and rarely in children less of ten years. [1][2]

A mucocele appears on imaging as an expansive lesion in an airless sinus, with thinning and sometimes erosion of its bony walls. Computerised tomographic scan (CT) has proven to be an excellent diagnostic tool and is essential in surgical planning. Magnetic resonance imaging (MRI) may provide additional information in the examination of the orbit and may be preferred imaging technique if other soft tissue tumours causing proptosis cannot be excluded. Differential diagnosis includes paranasal sinus carcinoma, Aspergillus infection, chronic infection or inverting papilloma. The most common treatment modality is extirpation of the mucocele, cranialization or exclusion of sinus and nasofrontal duct obliteration. [3][4]

\section{Case Report}

A 56 years old man, presented with a lump on the left and right forehead accompanied by a protruding left eye. This complaint has been experienced by the patient since 6 months and is getting worse so that the patient has difficulty opening his left eye. Patient with a history of craniectomy debridement surgery indicated for open depressed fracture due to an accident 12 years ago, then underwent a titanium mesh cranioplasty 11 years ago. There were no complaints of headache, vomiting and seizures. Full awareness GCS 15, motoric within normal limits, Vision ODS 6/6.

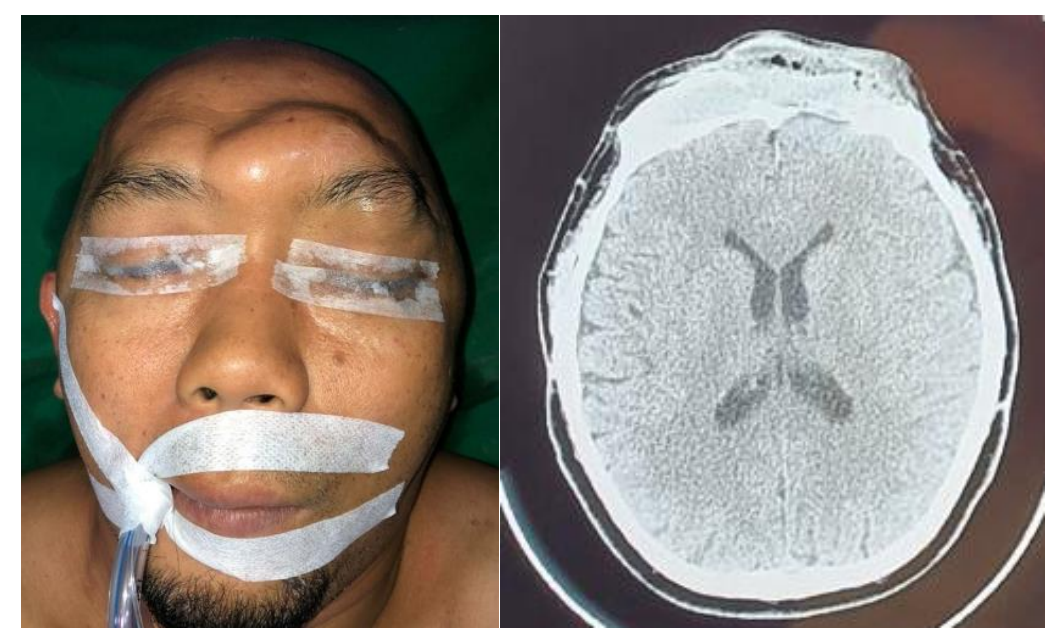

(A)

(B) 


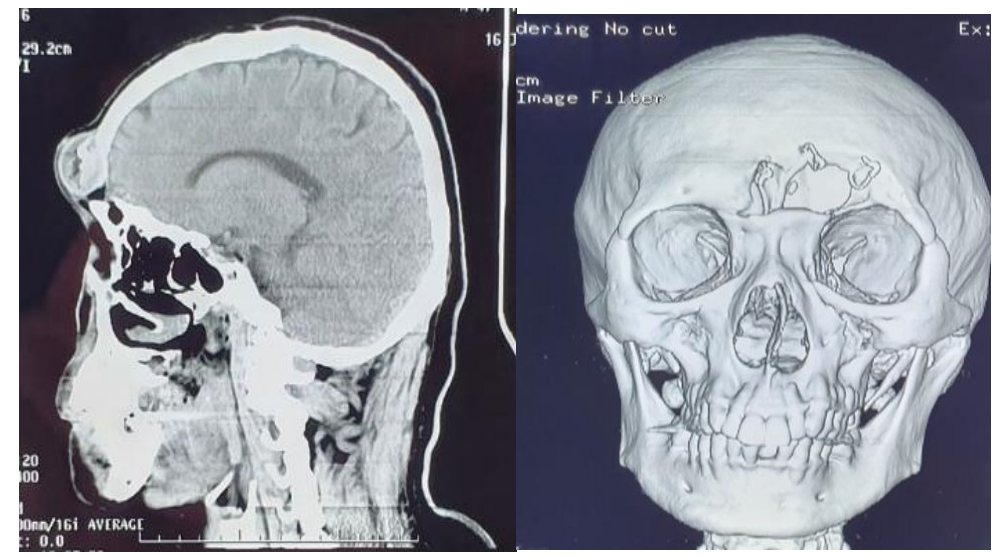

(C)

(D)

Figure 1.(A)Pre-operative (B) Head CT-Scan Axial View

(C) Coronal View (D) 3D view

From examination of the head CT scan revealed a solid mass lesion filling the left and right frontal sinuses. The lesion expands into the left orbital cavity. Bifrontal craniotomy was performed on the patient. During the operation, there was a lytic on the left frontal bone, found a mass that filled the left and right frontal sinuses that extended laterally, a greenish, soft and suctionable mass, evacuation of all the masses and infected bone was carried out.'

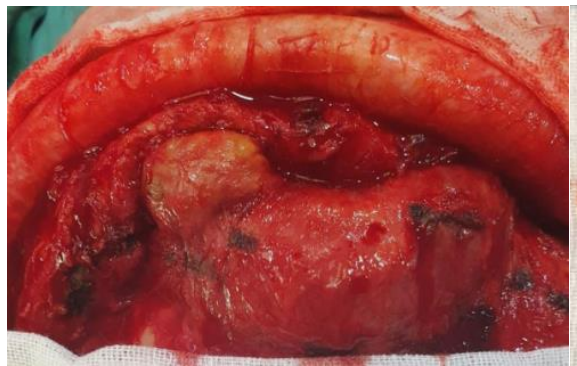

(A)

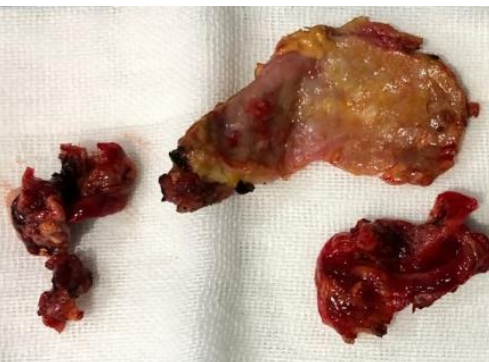

(B)

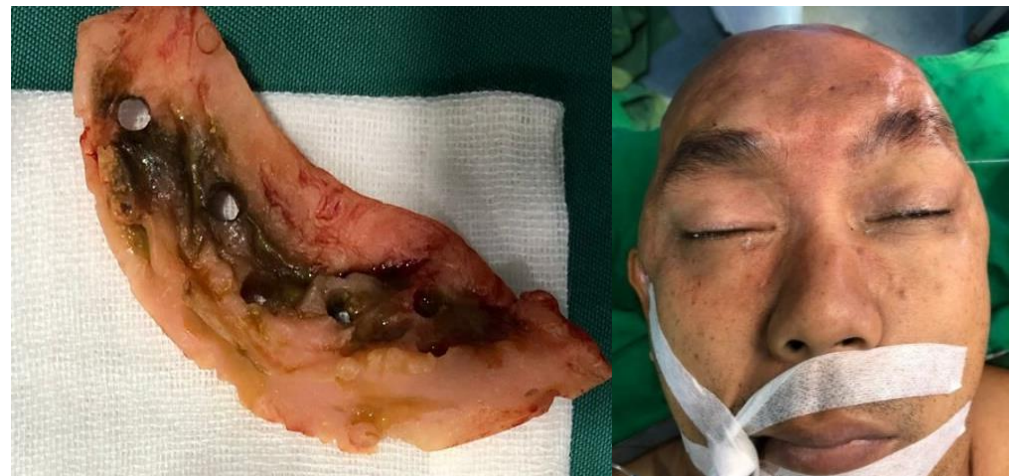

(C)

(D)

Figure 2. (A) Sinus frontalis sinistra bulging (B) Mucocele post excision (C) Infected Bone (D) Post operative 
Postoperatively patient with GCS of 15 , without neurological deficits. Complaints of lumps disappear and the eyelids can be opened normally.

\section{Discussion}

Frontal sinus cranialization, as first described by Donald and Bernstein, is often required when the integrity of the posterior table has been violated either by disease or by trauma. It has also been proposed as a method to prevent frontal sinus complications following frontal craniotomy. This technique involves removal of mucosa from the intact anterior table using both cutting and diamond burrs. The posterior table of the frontal sinus is removed using rongeurs, and the remaining portions are drilled flush with the anterior sinus walls, floor, and anterior skull base. The frontal recess is then occluded, followed by intracranial placement of the previously raised pericranial flap through a small defect in the anterior table. This procedure also have complication to develepong mucocele if not done in adequate procedures. [2][6][7]

Mucoceles are mucous-secreting expansive pseudocystic formations, and capable of expansion by virtue of a dynamic process of bone resorption and new bone formation [5]. They result from obstruction of a sinus ostium and frequently are related to a previous condition as chronic sinusitis, trauma, surgery or expansible lesion. With continued secretion and accumulation mucus, the increasing pressure causes atrophy or erosion of the bone of the sinus, allowing the mucocele to expand in the path of less resistance. This may be into the orbit, adjacent sinuses, nasal cavity, intracranial or through the skin; intracranial and orbital extension were demonstrated in this patient. [6]

Mucoceles are most commonly found in the frontal and ethmoidal sinuses, are infrequent in the sphenoid sinus and occur rarely in the maxillary sinuses. Proptosis is the most common presenting sign of a frontal mucocele, as in our case. Other clinical features include a mass in the upper medial quadrant of the orbit, pain, vertical diplopia, limited upward gaze, bifrontal headache and increasing tearing [7]. Occasionally, an aggressive mucocele will cause bone destruction, especially of the orbital wall and may then simulate a malignant neoplasm. In these cases, CT is particularly helpful to distinguish benign from malignant disease, because an expanding mucocele often preserves a thin fat plane between margins of the mucocele and the muscle cone. Other known complications of frontal mucoceles include erosion of the anterior wall, resulting in a tender fluctuant mass beneath the periosteum of the frontal bone. Erosion of the posterior wall may produce complications such as epidural abscess, meningitis, subdural empyema and brain abscess. Rarely, cranial 
nerve palsies may also occur. such as dysthyroid eye disease, retrobulbar orbital tumor, inflammatory pseudo tumour, sinus tumour and metastatic lesion. Progressive unilateral painless proptosis of gradual onset should make one suspicious of a mucocele involving the paranasal sinuses. This is especially so if there is accompanying diplopia, orbital or forehead pain and epiphora, which are frequently the presenting symptoms of mucoceles. [8]

The classic radiographic appearance of a mucocele is generalised thinning and expansion of the sinus walls and there may also be evidence of sinus disease as well as bony erosions. The mucocele usually appears homogenous and airless. CT scan is much better in delineating the extent of the lesion and its relations to other surrounding structures [8]. CT demonstrates an airless, mucoid density filled, expansile, non-enhancing sinus mass with gradual thinning and erosion of the bony margins. The extent of the bone destruction is also better appreciated on CT. Frontal sinus mucoceles tend to erode posterior wall because of its inherent thinness. MRI exhibits variable signal intensities on both T1- and T2- weighted images, depending on the state of hydration, protein content and viscosity of the contents of the mucocele. Contrast-enhanced MR imaging is useful in differentiating mucoceles from sinonasal tumours. Mucoceles characteristically reveal a thin peripheral linear enhancement with central low-signal intensity on T1 weighted images; and sinonasal tumours demonstrate diffuse enhancement. [9]

The definitive treatment of mucoceles is primarily surgical, but the mucoceles of the frontal sinus that extend into the orbits and the anterior cranial fossa can, in certain cases, be difficult to manage therapeutically and may lead to lethal complications [9]. Traditionally, frontal mucoceles were treated with external open obliterative procedure. Alternatively, functional endoscopic sinus surgery can be used to evacuate the mucocele, but this procedure become difficult if there is intracranial expansion [10]. An external approach, combined with endoscopic surgery, is suitable in frontal mucoceles, especially in distal sites and in case of recurrence. Pericranial flaps are reliable, thin, and supple and have a good arc of rotation and minimal donor site morbidity. The pericranium frontal flap obliterates the dead space and isolates the extradural space from contaminated facial cavities, diminishing the risk of infection of the anterior fossa [11].As in our case, we do the craniotomy, the external open approach has the advantages of: being a direct approach, with allows exposure of the entire sinus, provides complete obliteration of the sinus to prevent recurrence of the sinus disease, and prevents blind curettage of any exposed duramater (12).

A coronal incision, this approach was used in order to gain better access to the area of the lesion and in order to make reconstruction easier. The treatment was bifrontal craniotomy, 
removal of the posterior wall of the frontal sinus and extirpation of mucocele. Posteriorly, the reconstruction was made with pericranial flap. The prognosis for frontal sinus mucoceles is good with likelihood of cure, and a low incidence of recurrence. Although, for control of recurrences long-term follow-up is recommended [12]

\section{Conclusion}

A 56-year-old male patient was diagnosed with nasofrontal mucocele based on physical examination and imaging. Patients with a history of craniectomy debridement surgery before. Bifrontal craniotomy was performed on the patient. During the operation, there was a lytic on the left frontal bone, found a mass that filled the left and right frontal sinuses that extended laterally, a greenish, soft and suctionable mass, evacuation of all the masses and infected bone was carried out.

Meticulous removal of frontal sinus mucosa should be attempted in obliteration or cranialization of the frontal sinus. Physicians should be aware of the possibility of mucocele development after frontal craniotomy has been performed. Patients should undergo long-term surveillance to monitor for their formation.

Mucoceles arising in the frontal sinus frequently are clinically silent for a lot of time and often involve both the orbit and the anterior cranial fossa extensively. Frontal mucoceles are benign and curable, but early diagnosis and treatment of them is important. Open surgery remains a valid procedure in frontal mucoceles with orbital and/or intracranial extension and in cases where the district anatomy is unfavourable for a purely endonasal approach. 


\section{Refences}

[1]. Schramm VL, Maroon JC. Sinus complications of frontal craniotomy. Laryngoscope 1979;89:1436-1445.

[2]. Howarth WG. Mucocele and pyocele of the nasal accessory sinus. Lancet $1921 ; 2: 744-746$

[3].Constantinidis J, Steinhart H, Schwerdtfeger K, Zenk J, Iro H. Therapy of invasive mucoceles of the frontal sinus. Rhinology 2001;39:33-38.

[4].Yap SK, Aung T, Yap EY. Frontal sinus mucoceles causing proptosis. Two case reports. Ann Acad Med Singapore 1998;27:744-7.

[5]. Weitzel EK, Hollier LH, Calzada G, Manolidis S. Single stage management of complex fronto-orbital mucoceles. J Craniofac Surg 2002;13:739-45.

[6].Molteni G, Spinelli R, Panigatti S, Colombo L, Ronchi P. Voluminous frontoethmoidal mucocele with epidural involvement. Surgical treatment by coronal approach. Acta Otorhinolaryngol Ital 2003;23:185-90.

[7]. Gavioli C, Grasso DL, Carinci F, Amoroso C, Pastore A. Mucoceles of the frontal sinus: Clinical and therapeutical considerations. Minerva Stomatol 2002;51:385-90.

[8].Suri A, Mahapatra AK, Gaikwad S, Sarkar C. Giant mucoceles of the frontal sinus: a series and review. J Clin Neurosci 2004;11:214-8.

[9].Pia F, Aluffi P, Borello G. Frontal mucocele: open surgery. Acta Otorhinolaryngol Ital 1999;19:155-9.

[10]Delfini R, Missori P, Iannetti G, Ciappetta P, Cantore G. Mucoceles of the paranasal sinuses with intracranial and intraorbital extension: Report of 28 cases. Neurosurgery 1993;32:901-6.

[11]Lund VJ. Endoscopic management of paranasal sinus mucoceles. J Laryngol Otol 1998;112:36-40

[12] Weitzel EK, Hollier LH, Calzada G, Manolidis S. Single stage management of Complex fronto-orbital mucoceles. J Craniofac Surg 2002;13:739-45. 